(Switzerland) described refined thermistor cireuitry and instrumentation for the routine, precise determination of molecular weight using a sample weight of only $2.5 \mathrm{mgm}$. for a molecular weight of 500 . P. D. Garn and J. E. Kessler (United States) demonstrated that thermal analysis is feasible for sample weights less than $1 \mathrm{mgm}$. by the use of appropriately designed sample holders.

In the session on nucleonics, G. F. Morrison and J. F. Cosgrove (United States) described the application of neutron activation to the sensitive determination of the copper activator in single crystals of electroluminescent zinc sulphide weighing less than $500 \mu \mathrm{gm}$. B. A. Thompson (United States) considered how radiochemical techniques and especially neutron activation could be applied to the determination of the principal components, trace impurities and thickness of metal films a few angstroms in thickness and one square centimetre in area; such films are encountered in the performance and design of electronic devices, including vacuum tubes. M. T. Kelley (United States) reviewed the present status of the analysis of highly radioactive reactor fuels and spent products using liquid samples of 5-700 $\mu \mathrm{l}$.; coulometry, spectrophotometry, flame photometry, emission spectroscopy, titrimetry and radiometry, and density measurements are feasible by remote controlled, automatic or 'slave' manipulated operations. A. Holasek and co-workers (Austria) explained the difficulties and operational problems encountered in the synthesis of milligram amounts of fatty acids and esters labelled with carbon-14, and considered salient factors involved in the selection of suitable synthetic methods.

Chromatographic methods were the subject of many interesting papers. E. G. Wollish (United States) considered critically the accelerating interest in alumina and silicic acid for thin-layer chromatography in the separation and identification of organic compounds, including drugs. H. K. Mangold and co-workers (United States) exemplified how thinlayer chromatography acts as a valuable analytical and preparative tool in lipid chemistry. L. S. Ettre and W. Averill (United States) demonstrated how new gas chromatographic instrumentation using capillary (Golay) columns and ultra-sensitive detectors permit the direct analysis of trace components in samples of micro-size, and set forth criteria for the selection of methods and instrumentation. L. Wiesner and W.-J. Schmidt-Küster (Germany) described the gas-chromatographic performance of a new integrating counter-detector using a flow-type proportional counter containing a source of ionizing radiation and used with a suitable scaler. T. S. Ma and M. Gutterson (United States) recommended gas chromatography in the determination of several organic functional groups. S. Patton (United States) considered the identification of food flavours using a diversity of techniques including gas, paper and column chromatography, and the study of the dinitrophenylhydrazones of volatile keto-compounds. Paper chromatography was applied by B. Bobrański and L. Syper (Poland) to the identification of drugs containing unsaturated radicals following reaction with mercuric acetate. A. K. Dey and E. J. Singh (India) explained the behaviour and application of various complexing agents in the separation of metal ions by the Weisz ring-oven technique.

The contemporary position of small-scale methods in the teaching of chemistry at the secondary school and collegiate levels was assessed in a special session with speakers from Northern Ireland (C. L. Wilson, N. N. Gilpin), England (J. B. Headridge, H. Holness), and the United States (N. D. Cheronis, T. S. Ma and J. A. Kuck). A. Steyermark (United States) considered the training of chemists in industry in quantitative organic analysis.

When the Proceedings of this symposium become available as a single volume (Interscience, early 1962), the interested technologist will be able to ascertain the position of microchemistry and microanalysis for 1961 in the spectrum of scientific activity, and to comprehend the research trends that will yield the 'to-morrow' of these disciplines. In brief, microchemical and microanalytical research faces continuing challenges with ever smaller samples, ever more minute components, coupled with demands for adequate accuracy, precision and rapidity. Improved instrumentation, automation and new techniques and reagents, coupled with re-assessment of present methods and approaches, will energize further advances.

A. J. BARNARD, JUN. JoHN Y. STEEL

\title{
INSECT POLYMORPHISM
}

\begin{abstract}
A SYMPOSIUM on polymorphism in insects, organized by the Royal Entomological Society of London, was held at the Imperial College of Science and Technology, London, during September 21-22. The president, Prof. G. C. Varloy, was in the chair at the first meeting and expressed a hope that special attention would be directed to the agencies promoting and controlling this type of variation.

Prof. O. W. Richards, in a general introduction, stated that polymorphism is very widespread among the insects and occurs in all the major orders. The metamorphosis of insects, itself a mode of polymorphism, perhaps makes them especially prone to such variation. There are two principal types of polymorphism: the genetic type, where two or more genotypes co-exist in a state of balance, and the induced type, in which some variable environmental factor such as population-density, food, day-length
\end{abstract}

or temperature leads to the development of two or more kinds of individual in a species.

Dr. E. B. Ford, in an account of the theory of genetic polymorphism, stated that the standard examples are only special cases of a more general phenomenon which is often overlooked when the characters involved are not easily visible. This is the tendency for selection to favour the heterozygote over both homozygotes when, after some change in the environment, a rare major gene begins to spread. The favourable effects of the gene will tend to become dominant and the unfavourable ones recessive, and the heterozygote will have all the advantages and none of the disadvantages of the homozygotes. Where polymorphism results from the interaction of several genes, the whole complex is controlled by a supergene, the link between the units of which may be strengthened by a chromosome inversion. The original effects 
of the controlling mechanism are always liable to evolutionary change by selection of the remainder of the gene-complex.

Dr. P. M. Sheppard then reported on recent work on polymorphic mimetic swallowtail butterflies. Knowledge of the way in which mimicry arises and is maintained makes possible a number of predictions as to how the polymorphism should be controlled genetically. These predictions can then be compared with the genetics of the polymorphic mimics so far as these have been unravelled. The most striking results have been obtained in the African Papilio dardanus, in which several different forms of female, some mimetic and some not, correspond to a single type of male. Recently, methods have been devised for breeding the butterfly artificially in England and a genetic analysis combined with field data on population statistics show a good agreement with theoretical predictions.

Prof. T. Dobzhansky dealt with the dynamics of chromosomal polymorphism in Drosophila. In the giant chromosomes of the larval salivary glands one can recognize individuals which as a result of inversions of parts of chromosomes have different gene arrangements. Several chromosomal types may occur in one population and their proportion may be constant, vary seasonally, or over longer periods, or geographically. Such changes in proportion may directly demonstrate the action of natural selection and allow calculation of the selective advantages of the polymorphs. The mere order of the genes may itself be an element in the fitness of a polymorph, but perhaps more often an inversion by suppressing crossing-over preserves an arrangement which gives an advantage to heterozygotes. Several of the field phenomena can be reproduced in cage populations. The proportions of the polymorphs are easily modified by changes in the environment or in the genetic background. Crosses with stocks from widely separated sources have an unpredictable effect on the proportions of the inversions because of the large number of alternative backgrounds provided.

The next four contributions were concerned with familiar examples of non-genetic polymorphism, namely, in Hymenoptera, in termites, in aphids and in locusts. Prof. C. D. Michener dealt with the first topic and showed that in only a few bees and in ants is the worker caste sharply different from the queen. In most social bees and in wasps the differences are slight and overlapping, occurring mainly in behaviour and physiology, but in several ants the worker caste is itself polymorphic. In the least differentiated castes of some halictine bees, the slight differences arise in the adult stage but the castes of all other Hymenoptera, except perhaps Melipona, seem to depend on differences in nutrition, sometimes interacting with temperature. At some point in development, however, the caste becomes determined. In Pheidole, the workers produced from a queen's first batch of eggs are small, even if they were well fed; the type of worker is thus determined in the egg, but perhaps here through the nutrition of the queen which laid them. The selective advantages of the castes of advanced insect societies is clear but their evolution is anomalous, since the first step seems to be the reduction of fertility in some individuals. However, it appears that economy of labour and better defence of the nest make even the most primitive social organization (one queen and one worker in some halictine bees) more viable than isolated queens.
Dr. M. Lüscher gave an account of social control of polymorphism in termites, one of the most astounding of all forms of social regulation in animals. Termites may have up to seven different castes in most of which each sex is equally represented. The newly hatched insect is capable of developing into any of the castes by a series of moults. Some forms may also have regressive moults leading back to an earlier stage in the series. Caste determination mainly acts by directing moults in the required direction, but the workers may also reduce a caste to its correct proportions by selective cannibalism. The direction of moults is influenced by active substances or pheromones which are exchanged among members of the colony. This is most clearly established in the inhibition of the reproductive caste by members of the same caste already present. Lüscher has shown by fixing a queen in a screen so that her head is in one colony and her abdomen in another, and then varnishing over parts of her body, that the active substance comes from the anus. Complete inhibition, however, requires the presence of a male as well as a female reproductive, so that there seem to be two substances. Another one is probably involved in the inhibition of the soldier caste. There are also stimulating substances; more males, for example, being produced in a colony with a queen but no king than in one without either. Termites are only responsive to such influences for part of the time intervening between each moult. The substances probably act through an effect on the ductless glands of the insect. There may be as many as eight different pheromones, but none is as yet chemically determined.

Dr. A. D. Lees then described the clonal polymorphism of aphids, especially in Megoura viciae. Aphids may reproduce viviparously and parthenogenetically or be fertilized and lay eggs. They may be winged or apterous and there is more than one kind of wingless female. The full development of some of the morphal characters may appear gradually during several successive generations. Other morphs (for example, sexual-parthenogenetic) are strict alternatives. Controlling mechanisms are almost all environmental, acting through the endocrine system. Aphid reproduction is extremely precocious so that an embryo will already have ovulated at its deposition and it is thus necessary to determine which of the three generations is being affected. Males can be suppressed by suitable temperatures or in some species photoperiod but are otherwise produced in a characteristic percentage. Egg-laying females are produced at photoperiods of less than $15 \mathrm{hr}$. and temperatures of about $15^{\circ} \mathrm{C}$. Winged forms arise as a result of crowding and this is not an indirect effect acting through the host-plant. There is also an 'interval-timer' which does not allow sexual forms to be produced for some time (50-60 days at $15^{\circ} \mathrm{C}$.). By selecting the first and last members of each generation to start separate lines, it can be shown that the interval is a matter of days and not of generations.

Continuous polymorphism in locusts described by Dr. J. S. Kennedy is different from most of the other examples in that the morphological differences are relatively slight and intermediates are often commoner than the extremes. Yet not only the morphology but also almost every aspect of behaviour and physiology are somehow affected. Phase transformation is largely controlled by population-density, which may act directly or on the previous generation. Genetic 
make-up, temperature and other factors may also have some importance. The solitary phase may be regarded as more juvenile than the gregarious one, and recent experiments have shown that the prothoracic gland which normally determines a juvenile morphology persists throughout life in the solitaria although disappearing early in the adult gregaria. Dr. Kennedy suggested that solitaria is adapted to a 'soft' habitat where food is available without much movement. Gregaria is suited to a 'hard' habitat which can be exploited only by the continuous movement which is typical of the phase. The polymorphism as a whole allows the species to $\theta x-$ ploit a much wider habitat than one phase could alone.

Dr. J. H. Sang, speaking on the environmental control of mutant expression, dealt with a developmental problem. In the course of growth a gene may produce a graded response in an enzyme which nevertheless has a discontinuous effect on adult development. Thus the gene 'antennaless' in Drosophila determines 0,1 and 2 antennæ but nearly always perfect structures. This is because the action of the gene occurs during a very short but welldefined period, namely, between the seventieth and eightieth hours of larval growth. This is shown by transferring larvæ of various ages to a different culture medium, for the effect of the gene can largely be nullified by suitable food. Such examples suggest how polymorphism might be determined by either a genetic or an environmental switch mechanism, in which influences at the right state of growth may produce large and discontinuous changes in the adult.

Prof. V. B. Wigglesworth, in a tentative synthesis, drew analogies between the polymorphism of cells in different parts of the body, the polymorphic aspect of insect metamorphosis and the phenomena which the other speakers had discussed. He showed that there seemed to be a complete transition from variation in which genetic determination was almost absolute to that in which external environmental factors decided between several alternatives.

The animated discussion which followed the invited contributions did not bring forward many really new points but was ehiefly concerned with the relative importance of genetic and non-genetic factors in particular examples. Terminology was also discussed, and some speakers were unwilling to speak of polymorphism unless the variations were clearly discontinuous. The suggestion, however, was made that the best distinction was between situations in which there is selection for increased variability as opposed to the more normal case where deviations are discouraged.

O. W. RICHARDS

\section{MOLECULAR BIOLOGY AND POPULATION STRUCTURE}

\begin{abstract}
A SYMPOSIUM on "Molecular Biology and Population Structure" was held at Norwich on the afternoon of September 4 at a joint session of Sections D (Zoology) and K (Botany) of the British Association for the Advancement of Science. The aim of the symposium was not so much to present new discoveries as to demonstrate to the audience the interrelations of recent discoveries in these fields, and to discuss some of their implications.

The symposium was opened by Dr. T. Alderson (British Empire Cancer Campaign), who described what we know of the structure of deoxyribonucleic acid (DNA) and brought out some of the evidence that genetic differences as we observe them are the resultant effects of differences of DNA molecules. Dr. J. A. Pateman (University of Cambridge) followed with a discussion of the evidence that gene differences have their effects by influencing the structure of protein molecules, though in discussing, at the end of his review, the recently discovered class of genes that appear to have the capacity of "turning on and off" whole metabolic sequences, he pointed out that there is evidence that these do not operate by producing proteins. These new regulator genes may well prove of major importance for our understanding of differentiation. Prof. H. Grüneberg (University College, London) then made the connexion between enzymes and more conventional characters by describing some beautiful examples, from his work with mice, which showed how the little invisible differences in chemical structure may be magnified during embryological development into the larger more obvious differences with which the study of genetics began.
\end{abstract}

At this point the symposium turned to what at first sight was another topic altogether, when Prof. J. M. Thoday (University of Cambridge) described how a variety of genes may be maintained in a population if that population were exposed to a variety of demands. Dr. P. M. Sheppard (University of Liverpool) then went on to discuss some of the evidence, illustrated from his work on butterflies, that the variety of genetic types in a population are not only directly adapted to the variety of conditions which the members of the population can exploit to promote survival, but must also be adapted to one another. The population is an integrated unit, and over the generations the genetic contents of different components of the population are judged not only by their individual success but also by the degree to which they co-operate with one another when brought together in sexual reproduction. The chromosomes of a population have not only to have genetic properties that are to be understood in terms of the needs of individuals but also other genetic properties only to be understood in terms of the population as a whole. Among other things the chromosomes of different individuals have to be coadapted to one another if the population is to function successfully over the generations.

Dr. C. A. Clarke (Royal Infirmary, Liverpool) followed. Dr. Sheppard with a little of the evidence that all these principles apply to man just as much as to Drosophila, or butterflies.

Prof. J. M. Thoday gave the final paper. $\mathrm{He}_{\mathrm{e}}$ pointed out that the two most striking discoveries of modern genetics had been brought together in this symposium.

The first discovery concerns the molecular nature of the genetic material. It has, of course, long been supposed that genes are molecules of some sort or another, but the precise knowledge of their molecular nature we are now acquiring is one of the most spectacular achievements of biology. 